\title{
128. Resting and Action Potentials in the Region of the Atrioventricular Node
}

\author{
By Toyomi Sano, Minoru Tasaki, Masaru Ono, Hiromichi \\ Tsuchinashi, Noriyuki Takayama, and Takio Shimamoto \\ Department of Clinical Physiology, Tokyo Medical and Dental University \\ (Comm. by T. Furuhata, M.J.A., Oct. 13, 1958)
}

There have been many reports on resting and action potentials of the Purkinje fiber and of the cardiac cell at the sinoatrial node, at the atrium, and at the ventricle studied by the microelectrode method. As for the resting and action potentials of the atrioventricular node, Hoffman et al. ${ }^{\text {') }}$ described in an article in 'Letters to the Editor' in 'Nature' that the action potential of the atrioventricular node was similar in voltage-time course to that of the sinoatrial node, its rising velocity was low, and diastolic depolarization was prominent, and Matsuda et $a .^{2}{ }^{2}$ reported that they were small, and its action potential had a peculiar configuration beginning with a step before its steepest rise. But no systematic study on those in the region of the atrioventricular node including anatomical examination seems to have been reported up to this time. This is the first report of a series of such studies.

METHOD. Dogs of a great variety in age and weight were anesthetized by intravenous injection of thiopental sodium. Under artificial respiration their hearts were isolated. By cutting the anterior wall of the right venticle transversely and lifting the cut end, the septal and anterior cusps of the tricuspid valve, the coronary orifice, and the fossa ovalis were exposed. The so-called Koch's triangle ${ }^{3)}$ and the results of preliminary anatomical examination with other dog hearts helped in locating the atrioventricular node. In actual experiments the characteristic configuration of the action potential described later was guidance for identification. The details on the muscle chamber with 100 to $200 \mathrm{cc}$ of aerated Tyrode solution kept at $37^{\circ} \mathrm{C}$ and the perfusion system were described elsewhere. ${ }^{4)}$ Stimulating cathode, silver wire of $100 \mu$ in diameter insulated except for its tip, was placed in the right atrium close to the orifice of the coronary sinus, and another electrode, a gauze swab, was placed at a convenient place on the isolated muscle strip. Stimulus current was square pulses of 60 per minute. Stimulation was usually synchronized with the sweep

Addendum. This manuscript was handed for communication in July 1958. Slightly before reading this galley-proof reference 2 was published. Therefore, only the related parts were corrected. 
circuit of the cathode ray oscilloscope.

Our method of microelectrode using glass capillary electrodes, the external tip diameter of which was about $0.5 \mu$, and the recording system were also reported elsewhere. ${ }^{5)}$

RESUlTs. The action potential in the normal state but driven electrically. The configuration of the action potential in the region of the atrioventricular node was characteristically different from that in other regions of the heart. The most peculiar finding was that the action potential had a step-like part at the beginning of its rising limb which gradually or more suddenly proceeded to its steepest rise. Therefore the action potential here began with upward convex curve, having an extra inflection point at its top, whereas that in other regions with upward concave curve. The height of the transitional point between this step and the steepest rise varied greatly, but usually ranged from -23 to $-48 \mathrm{mV}$. The duration of the step also varied remarkably, but was usually from 0.05 to 0.11 seconds. Furthermore, the steepest rise of the action potential here was less steeper than that in other regions. The other parts of the action potential resembled in configuration to the action potential from the sinoatrial node. The action potential here was often followed by hyperpolarisation.

Other characteristics in this region were smallness of action potential, resting potential, and overshoot in magnitude. Summarizing 105 impalements with $25 \mathrm{dogs}$, the average resting potential was $53 \mathrm{mV}$, with a standard deviation of $\pm 7 \mathrm{mV}$. The action potential had an average amplitude of $58 \mathrm{mV}$ and the overshoot one of $5 \mathrm{mV}$ (S.D. $\pm 5 \mathrm{mV}$ ). These values were definitely smaller than those obtained from our measurements with atrial and ventricular cells. ${ }^{6)}$ The whole duration of the action potential was $354 \mathrm{msec}$ in average (S.D. $\pm 47 \mathrm{msec}$ ) under constant stimulating frequency of 60 per minute.

Regional difference of the action potential. The spot where the above-mentioned characteristics were observed had a very limited extension, about $1 \mathrm{~mm}$ in size. Although this spot was always within the Koch's triangle and could be located usually with ease after some experience, some individual difference was noticed in its position, and occasionally it could not be located by any means within short time. In the region surrounding this spot a transitional type of action potential was observed. This action potential was usually similar in shape with that from the sinoatrial node and smaller in size than the atrial action potential, having intermediate values.

Within this spot with characteristic features some regional difference in the action potential could be noticed from point to point. Main difference seems to be due to conduction delay at each point. There was also some individual difference in the topography of such points. An example of the regional difference within this spot and in the surrounding region was shown in Fig. 1. 


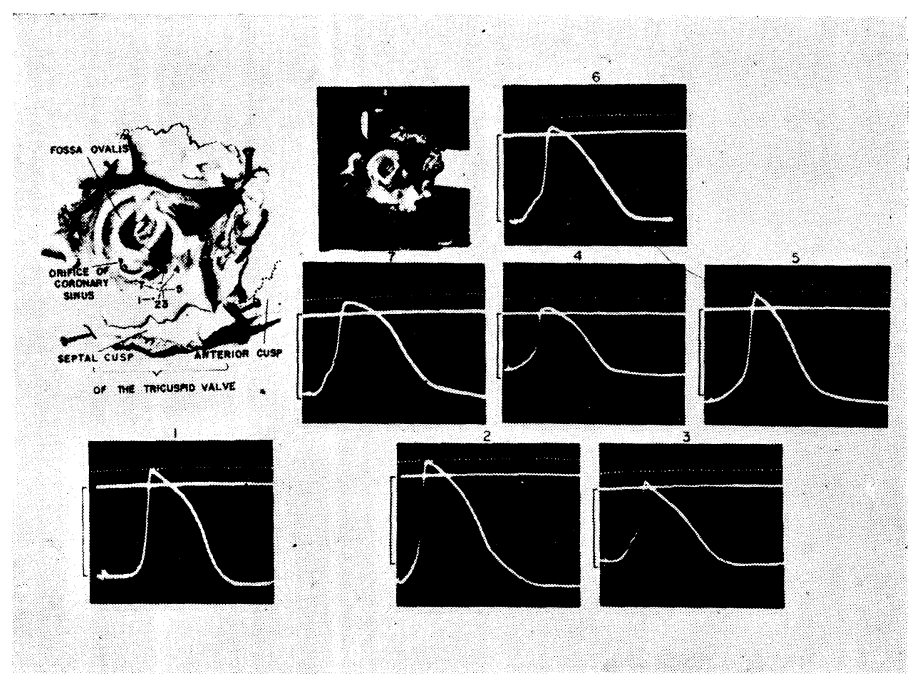

Fig. 1. Regional difference of resting and action potentials in the region of the atrioventricular node. Stimulating cathode was placed on the left side of the orifice of the coronary sinus and another electrode $1 \mathrm{~cm}$ upward from the left end of the septal cusp of the tricuspid valve. A pin was inserted at the center of the region where specific features were observed, as is seen in the photograph of the preparation. The number of each photograph indicates the spot from which the photograph was taken, the numbers in the picture at the left upper corner corresponding to those of the photographs. Amplification was the same in all the numbered figures except in 3 and 4 which were of smaller amplification. This is shown by the bar on the left side of each photograph which denotes the zero level and $-50 \mathrm{mV}$. Time marks at intervals of $10 \mathrm{msec}$. The action potential from 1 is close to the atrial action potential in shape.

The action potential when the region of the atrioventricular node gained spontaneous activity. Frequently it became difficult to drive

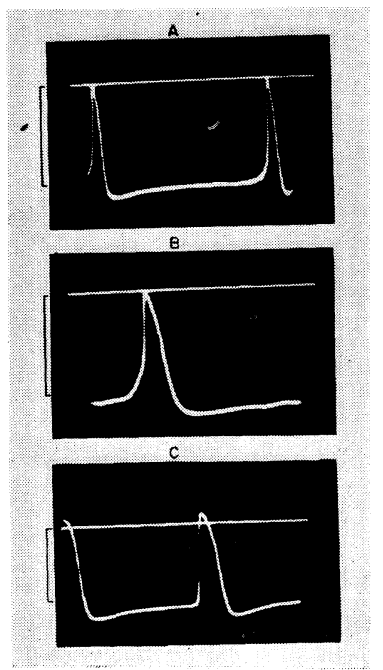

Fig. 2. Action potentials in the region of the atrioventricular node in spontaneous activity. Slow diastolic depolarization is noticed in A. That the step of the action potential in this region can be distinguished from it is shown in B which was obtained from the similar region with faster sweep. Furthermore, records from the surrounding region, which are regarded as of a transitional type, showed also slow diastolic depolarization as is seen in $\mathrm{C}$, but these action potentials have no step. Time marks at intervals of 100 msec. The bars on the left side of the photographs denote 0 and $-50 \mathrm{mV}$. 
the muscle strip by electrical stimuli because this region gained spontaneous activity. In this case slow diastolic depolarization appeared, as was noticed in the sinoatrial node, instead of constant resting potential between two successive action potentials, as was seen in the driven condition. Then, not only this spot with characteristic features, but also the surrounding, more extensive region with a transitional type showed such slow diastolic depolarization (Fig. 2).

Atrioventricular block. Under some abnormal conditions it was noticed that the duration of the step prolonged progressively in successive

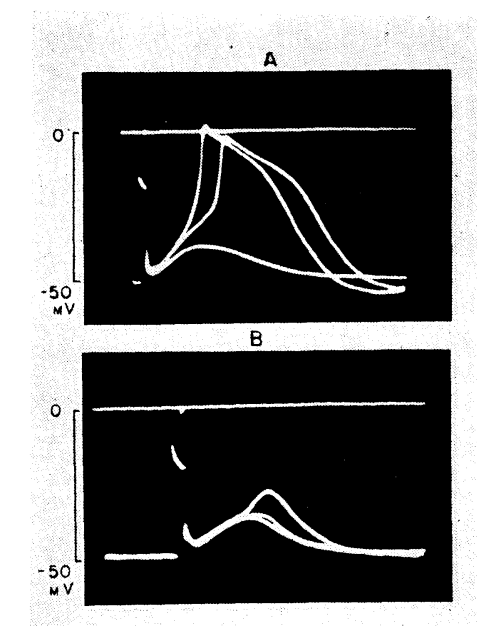

Fig. 3. Membrane potentials in the region of the atrioventricular node in the case of atrioventricular block. Successive three beats were photographed. Time marks at intervals of $100 \mathrm{msec}$. See text. beats until finally only a small localized depolarization was observed (Fig. 3A). Repetition of this sequence of events corresponds to the so-called Wenckebach's phenomenon. In complete atrioventricular block or incomplete block with occasional ventricular capture only localized depolarization was observed for a while (Fig. 3B).

Effects of various drugs. The effects of $l$-noradrenaline were similar with those of adrenaline. ${ }^{2)}$ Immediately after two drops of $0.2 \% \quad l$-noradrenaline were added close to the impaled spot in $110 \mathrm{cc}$ of the Tyrode solution, the rate of rise of the step was increased. The duration of the step shortened and the steepest rise and other parts of the action potential advanced, as is shown in Fig. 4:1. Procaine amide and quinidine showed effects opposite to the above. When two drops of $10 \%$ procaine amide were added, the rate of rise of the step was decreased, lengthening the duration of the step, which resulted in retroceding of the chief part of the action potential (Fig. 4:3). The effects of digitalis glycosides were similar with those of procaine amide or quinidine in this respect. However, an addition of a few drops of $0.0075 \%$ G-strophanthin caused no observable change. In an example shown in Fig. 4:2 slight retroceding was first observed after its successive addition of 8 drops in total. With its further addition loss of plateau and slight decrease of the action potential appeared besides until it became much smaller. All the changes mentioned above were proved to be reversible.

Histological identification of the cells showing such characteristic features. Some of the cells showing the above-mentioned characteristic 


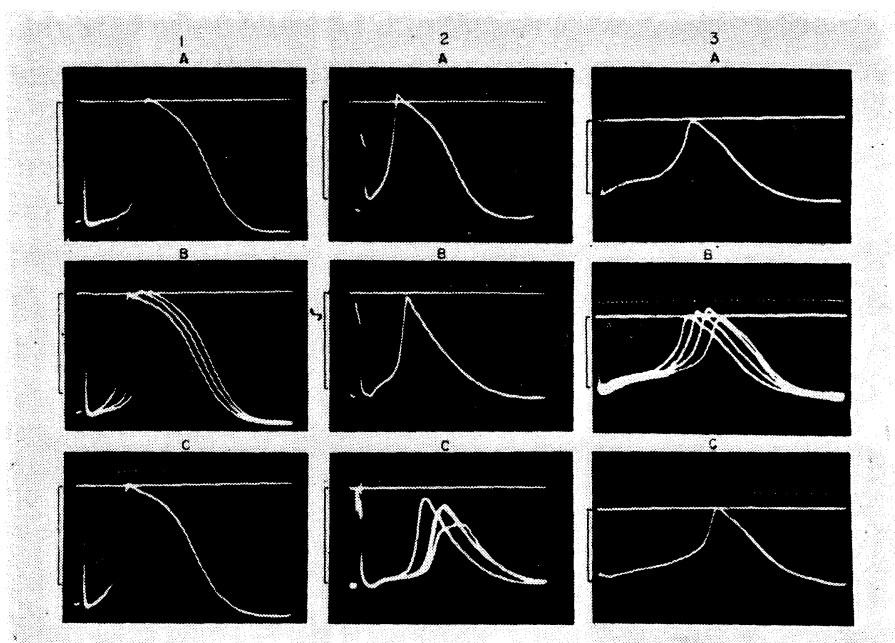

Fig. 4. Effects of noradrenaline (1), strophanthin (2), and procaine amide (3) on resting and action potentials in the region of the atrioventricular node. A: Control; 1B: Changes immediately after addition of 2 drops of $0.2 \% l$-noradrenaline; 1C: Final stage; $2 \mathrm{~B}$ : After successive addition of 10 drops of $0.0075 \%$ G-strophanthin in total; $2 \mathrm{C}$ : After its addition of other 4 drops; 3B: Immediately after addition of 2 drops of $10 \%$ procaine amide; 3C: Final stage. Time marks in 1 and 3 at intervals of 10 msec and in 2 at intervals of $100 \mathrm{msec}$. The bars on the left side of each photograph denote 0 and $-50 \mathrm{~m} \mathrm{~V}$.

features were examined histologically to determine their localization employing a special staining technique, ${ }^{8)}$ i.e. driving out ferricyanide in the microelectrode by passing electric current and adding ferrous chloride thereafter. Thus it was confirmed that some of them were actually the cells of the atrioventricular node or of the special junctional tissue surrounding it. Details of such histological study will be reported later.

Discussion. Hoffman and others ${ }^{1)}$ do not seem to have found clearly characteristic features at the beginning of the action potential in this region, judging from their report only. Their experiments were performed with rabbit hearts. Although not included in this report, we found the same features with rabbits. But whether or not this peculiar type of resting and action potentials originates actually from the atrioventricular node itself, as was suggested by Matsuda ${ }^{2)}$ without histological confirmation, had left some doubt. The impaled spots were approximately consistent with the anatomical location of the atrioventricular node. The chief doubt had lain in the depth of its location. As Asami ${ }^{7)}$ reported on whale hearts and as we ascertained by our own examination on dog hearts under his guidance, the atrioventricular node lies in the deeper zone under atrial muscle fibers. But not rarely it was experienced that the characteristic features could be observed before the microelectrode was inserted so far. It was first confirmed 
by us that such characteristic features originated actually from the special junctional tissue. Whether or not there are any other cells which show similar resting and action potentials is under study in this laboratory.

Furthermore, various properties, functionally ascribed to the atrioventricular node and its vicinity, seem to find their counterparts in the observed characteristics. For instance, spontaneous activity showing diastolic depolarisation was often observed in this region, although it was found in a more extensive region. Shortening of the PR interval of the electrocardiogram by adrenaline and noradrenaline or its prolongation by digitalis, quinidine, and procaine amide is consistent with shortening or prolongation of duration of the step by these drugs.

The counterparts of various atrioventricular blocks were found to be graded, localized depolarization in this region. From Fig. 3 and from other experiments it is assumed that the step of the action potential here corresponds to such graded depolarization.

SUMmARY. The resting and action potentials in the region of the atrioventricular node were studied by the intracellular microelectrode method. The action potential here had a characteristic configuration. Different from that in other regions it had a step-like part at the beginning of its rising limb. Resting potential, action potential, and overshoot were all remarkably smaller than those in other regions. The average resting potential, action potential, and overshoot were 53, 58, and $5 \mathrm{mV}$ respectively. The spot where such characteristic action potential was obtained had a very limited extension, about $1 \mathrm{~mm}$ in size. It was histologically confirmed that this action potential originated actually from the cells of the special junctional tissue. When this region gained spontaneous activity, slow diastolic depolarization was observed. When $l$-noradrenaline was added to the Tyrode solution, the rate of rise of the step was increased, the duration of the step shortened, and other parts of the action potential advanced. Procaine amide, quinidine, and digitalis glycosides had opposite effects, resulting in retroceding of the chief parts of the action potential. These changes were completely reversible.

\section{References}

1) Hoffman, B. F., Paes de Carvalho, A., and Carlos de Mello, W.,: Nature, 181, no. 4601, 66 (1958).

2) Matsuda, K., Hoshi, T., and Kameyama, S.,: Tohoku J. Exp. Med., 68, 8, 16 (1958).

3) Koch, W.,: Ziegler's Beiträge, 42, 203 (1907).

4) Sano, T., Takayama, N., and Shimamoto, T.,: Circulation Research (in press).

5) Sano, T., Ono, M., and Shimamoto, T.,: Circulation Research, 4, 444 (1956).

6) Sano, T., Tasaki, M., Tsuchihashi, H., Ono, M., and Shimamoto, T.,: Japanese Circulation Journal (in press).

7) Asami, I.,: Acta Anatomica Nipponica, 33, 1 (1958).

8) Tomita, T., Murakami, M., Sato, Y., and Hashimoto, Y.,: Jap. J. Physiol. (in press). 